\title{
Deriving robust unfitted finite element methods from augmented Lagrangian formulations
}

\author{
Erik Burman ${ }^{\dagger}$ and Peter Hansbo* \\ ${ }^{\dagger}$ Department of Mathematics, University College London, London, UK-WC1E 6BT, United Kingdom \\ ${ }^{\star}$ Department of Mechanical Engineering, Jönköping University, SE-55111 Jönköping, Sweden
}

\begin{abstract}
In this paper we will discuss different coupling methods suitable for use in the framework of the recently introduced CutFEM paradigm, cf. Burman et al. [6]. In particular we will consider mortaring using Lagrange multipliers on the one hand and Nitsche's method on the other. For simplicity we will first discuss these method in the setting of uncut meshes, and end with some comments on the extension to CutFEM. We will, for comparison, discuss some different types of problems such as high contrast problems and problems with stiff coupling or adhesive contact. We will review some of the existing methods for these problems and propose some alternative methods resulting from crossovers from the Lagrange multiplier framework to Nitsche's method and vice versa.
\end{abstract}

\section{Introduction}

Recently there has been increased interest in unfitted finite element for the imposition of boundary conditions or more generally for the coupling of physical systems over an interface. The unfitted discretization is then made as independent as possible of the geometric description of interfaces and domain boundaries in order to minimize the complexity of mesh generation. One such method is the Cut Finite Element Method (CutFEM) [6], the goal of which is to retain the accuracy and robustness of a standard finite element method. To reach this aim, stabilization techniques are applied to make both the accuracy of the approximation and the system condition number independent of the mesh/boundary intersection and physical parameters. Thanks to this robustness of the discretization, powerful linear algebra techniques developed for finite element methods are made available for solving the linear systems obtained by the CutFEM discretization.

In the CutFEM approach the boundary of-or interfaces in-a given domain is represented on a background grid, e.g., using a level set function, and the background grid is also used to discretize the governing partial differential equations. CutFEM builds on a general finite element formulation for the approximation of partial differential equations, in the bulk and on surfaces (interfaces or boundaries), that can handle elements of complex shape and where boundary and interface conditions are built into the discrete formulation. CutFEM requires only a low-quality, even non-conforming, surface mesh representations of the computational geometry, thus mitigating mesh generation work.

Unfitted methods typically use either Lagrange multipliers or Nitsche's method for the mortaring on interfaces or boundaries and it has been shown that Nitsche's method can be derived from a stabilized Lagrange muliplier method, due to Barbosa and Hughes [2] after static condensation of the multiplier [27] 23], cf. Section 2.2.

Another, fluctuation based, stabilization of the Lagrange multiplier was proposed in [10] and further developed in the works [9, 5, 3] and also [25] of this collection. For this type of methods the multiplier typically may not be eliminated, which can be a disadvantage; however, in some situations it is desirable to define the multiplier on a different space anyway.

Regardless of the relative virtues of the two approaches it is interesting to compare them and see how developments for one type of methods can be exploited in the context of the other. Similarly as in [27] we will here be interested in deriving methods using Lagrange multipliers and then recover the associated Nitsche method by formal elimination of the multiplier. However in our case we will base the discussion 
on the concept of augmented Lagrangian methods, which has recently been successfully applied in the context of contact problems using Nitsche's method [16, 17] and Lagrange multipliers [12].

The idea behind the augmented Lagrangian is to add a least squares term on the constraint to the Lagrangian functional of the constrained optimization problem on the discrete level. Provided this modification is not too strong it is expected to improve the conditioning as well as improving the control of the constraint compared to the case where only the multiplier is used to enforce the constraint. For early work on augmented Lagrangian methods in computational methods for partial differential equations we refer to Glowinski and le Tallec [20] or Fortin and Glowinski [19]. However, in cases where the problem depends on physical parameters that have some singular behavior, the augmented Lagrangian can lead to a severely ill-conditioned problem. This is typically the case for problems with high contrast in the diffusivity or strongly bonded adhesive problems. The ill-conditioning results from the fact that in the singular limit the least squares term on the constraint blows up, leading to ill-conditioning and also locking, unless the mesh-size is small enough to resolve the singularity. In many problems such a resolution is unfeasible and it is then useful to relax the size of the least squares term. This idea has been implicitly used in a number of works, starting with the paper [22] on debonding problems using Nitsche's method on unfitted meshes and then further developed for free flow porous media coupling in [8] and boundary conditions in [24]. A recent inventive use of this framework was proposed in [18] where it was applied to coupling schemes in fluid-structure interaction. All of these works are concerned with Nitsche type formulations.

Nevertheless there seems to have been no attempts at exploring these ideas directly in the framework of augmented Lagrangian methods. Our main objective in this paper is to study some model problems, exhibiting the typical parameter dependent behaviour, in the framework of augmented Lagrangian methods.

The idea is to first revisit the imposition of boundary conditions with Lagrange multipliers and Nitsche's method. Then we consider three different situations of domain decomposition. Here we assume that the problem is set on a domain $\Omega$ with two systems, defined in the subdomains $\Omega_{1}$ and $\Omega_{2}$, that are coupled over a smooth boundary $\Gamma$. For simplicity we consider Poisson type problems and do not discretize the boundary $\Gamma$, that is, we consider the semi-discretized setting. The discussion can easily be extended to for instance compressible elasticity. We will consider the following three model problems:

1. general boundary conditions;

2. Poisson's equation with high contrast in the diffusion coefficient;

3. debonding and adhesive contact.

First, in Section 2, we will recall, for an uncut mesh with Dirichlet boundary conditions, how least squares stabilized Lagrange multiplier methods lead to Nitsche's method following [27], and show how, alternatively, the augmented Lagrangian approach leads to the same formulation. Then, in Sections 3 5 we consider the three different model problems in the augmented Lagrangian framework and derive robust Nitsche methods as well as robust Lagrange multiplier methods, still formulated on uncut meshes. Some of the proposed methods appear to be new, whereas others are known in the literature and we will discuss existing results for the methods and without going into technical details we will speculate on what results are likely to carry over to the cases considered herein. In Section 6 we round off with some remarks considering the extension to cut finite element meshes, in particular with respect to stabilization of the discrete system, and, finally, in Section 7 we give a numerical example for one of the model problems.

\section{Derivations of Nitsche's method from Lagrange multipli- ers}

\subsection{Model problem}

Let us first consider the typical Poisson model problem of finding $u$ such that

$$
-\Delta u=f \text { in } \Omega, \quad u=g \text { on } \Gamma:=\partial \Omega,
$$

where $\Omega$ is a bounded domain in two or three space dimensions, with outward pointing normal $n$, and $f$ and $g$ are given functions. For simplicity, we shall assume that $\Omega$ is polyhedral (polygonal). The typical 
way of prescribing $u=g$ on the boundary is to pose the problem (1) as a minimization problem with side conditions and seek stationary points to the functional

$$
\mathscr{L}(v, \mu):=\frac{1}{2} a(v, v)-\langle\mu, v-g\rangle_{\Gamma}-(f, v)_{\Omega},
$$

where

$$
(f, v)_{\Omega}:=\int_{\Omega} f v d \Omega, a(u, v):=\int_{\Omega} \nabla u \cdot \nabla v d \Omega,\langle\mu, v-g\rangle_{\Gamma}:=\int_{\Gamma} \mu(v-g) d s .
$$

The stationary points are given by finding $(u, \lambda) \in H^{1}(\Omega) \times H^{-1 / 2}(\Gamma)$ such that

$$
\begin{gathered}
a(u, v)-\langle\lambda, v\rangle_{\Gamma}=(f, v) \quad \forall v \in H^{1}(\Omega), \\
\langle\mu, u\rangle_{\Gamma}=\langle\mu, g\rangle_{\Gamma} \quad \forall \mu \in H^{-1 / 2}(\Gamma) .
\end{gathered}
$$

As is well known, the discretization of this problem requires balancing of the discrete spaces for the multiplier $\lambda$ and the primal solution $u$ in order for the method to be stable, for examples cf. [4]. In the following we shall not consider balanced (inf-sup stable) discrete methods but instead focus on stabilized methods.

\subsection{Nitsche's method as a stabilized multiplier method}

Formally, the Lagrange multiplier in (3) is given by $\lambda=\partial_{n} u$, where $\partial_{n} v:=n \cdot \nabla v$, and a well known stabilization method [2] for the discretization of [3]- (4) to is to add a term penalizing the difference between the discrete multiplier and the discrete normal derivative of the primal solution. To this end, we assume that $\mathscr{T}_{h}$ is a conforming shape regular meshe on $\Omega$, consisting of triangles $T$ and define $V_{h}$ as the space of $H^{1}$-conforming piecewise polynomial functions on $\mathscr{T}$,

$$
V_{h}:=\left\{v_{h} \in H^{1}(\Omega):\left.v_{h}\right|_{T} \in \mathbb{P}_{k}(T), \forall T \in \mathscr{T}\right\}, \quad \text { for } k \geq 1 .
$$

As discrete space for the multiplier, we define the trace mesh on $\Gamma$ as the set $\mathscr{F}_{h}$ of element faces $F$ on $\Gamma$ and set

$$
\Lambda_{h}:=\left\{q_{h} \in L_{2}(\Gamma):\left.q_{h}\right|_{F} \in \mathbb{P}_{l}(F), \forall F \in \mathscr{F}\right\}, \quad \text { for } l \geq 0 .
$$

Then we seek $\left(u_{h}, \lambda_{h}\right) \in V_{h} \times \Lambda_{h}$ such that

$$
a\left(u_{h}, v\right)-\left\langle\lambda_{h}, v\right\rangle_{\Gamma}-\left\langle\mu, u_{h}\right\rangle_{\Gamma}-\frac{1}{\gamma_{0}}\left\langle h\left(\lambda_{h}-\partial_{n} u_{h}\right), \mu-\partial_{n} v\right\rangle_{\Gamma}=(f, v)-\langle\mu, g\rangle_{\Gamma}
$$

for all $(v, \mu) \in V_{h} \times \Lambda_{h}$. Here $h$ is the meshsize of the trace mesh on $\Gamma$, interpreted as a piecewise constant function along $\Gamma$ and $\gamma_{0}$ is a number to be chosen sufficiently large to obtain a stable method. Following Stenberg [27] we now let $P_{h}: L_{2}(\Gamma) \rightarrow \Lambda_{h}$ denote the $L_{2}$-projection, and considering $\Lambda_{h}$ to be a space of discontinuous discrete multipliers, continuous inside each face $F$ of the Lagrange multiplier mesh on $\Gamma$, we can eliminate the multiplier from (5):

$$
\left.\lambda_{h}\right|_{E}=\left.P_{h} \partial_{n} u_{h}\right|_{E}-\left.\gamma_{0} h^{-1} P_{h}\left(u_{h}-g\right)\right|_{E} \quad \forall E .
$$

Now considering the limiting case of $\Lambda_{h} \rightarrow L_{2}(\Gamma)$ we see that

$$
\lambda_{h} \rightarrow \partial_{n} u_{h}-\gamma_{0} h^{-1}\left(u_{h}-g\right)
$$

and we can reintroduce this multiplier into our stabilized method, replacing also $\mu$ by $\partial_{n} v-\gamma_{0} h^{-1} v_{h}$, to obtain the problem of finding $u_{h} \in V_{h}$ such that

$$
a\left(u_{h}, v\right)-\left\langle\partial_{n} u_{h}, v\right\rangle_{\Gamma}-\left\langle\partial_{n} v_{h}, u_{h}\right\rangle_{\Gamma}+\gamma_{0}\left\langle h^{-1} u_{h}, v\right\rangle_{\Gamma}=L(v) \quad \forall v \in V_{h},
$$

where

$$
L(v):=(f, v)-\left\langle\partial_{n} v, g\right\rangle_{\Gamma}+\gamma_{0}\left\langle h^{-1} v, g\right\rangle_{\Gamma}
$$

which is Nitsche's method [26]. 


\subsection{Nitsche's method as an augmented Lagrangian method}

The other approach to deriving Nitsche's method from Lagrange multipliers is more in the vein of Nitsche' original paper [26] where the method was derived from a discrete minimization problem without multipliers. The Lagrangian in (2) on the discrete spaces is augmented by a penalty term mutliplied by $\gamma \in \mathbb{R}^{+}$so that we seek stationary points to

$$
\mathscr{L}(v, \mu):=\frac{1}{2} a(v, v)-\langle\mu, v-g\rangle_{\Gamma}+\frac{1}{2}\left\|\gamma^{1 / 2}(v-g)\right\|_{\Gamma}^{2}-(f, v)_{\Omega},
$$

leading to the problem of finding $(u, \lambda) \in H^{1}(\Omega) \times H^{-1 / 2}(\Gamma)$ such that

$$
a(u, v)-\langle\lambda, v\rangle_{\Gamma}+\langle\gamma u, v\rangle_{\Gamma}-\langle\mu, u\rangle_{\Gamma}=(f, v)_{\Omega}+\langle\gamma g, v\rangle_{\Gamma}-\langle\mu, g\rangle_{\Gamma}
$$

for all $(v, \mu) \in H^{1}(\Omega) \times H^{-1 / 2}(\Gamma)$. Choosing now in the discrete case $\lambda_{h}:=\partial_{n} u_{h}, \mu=\partial_{n} v$, and $\gamma=$ $\gamma_{0} h^{-1}$ we recover (7). It should be noted that augmented Lagrangian methods are not in general inf-sup stable; typically an unstable method remains unstable and the augmentation rather serves the purpose of strengthening a method where the side condition is too weakly enforced. It is therefore rather remarkable that in the particular case where the discrete multiplier is replaced by the discrete normal derivative, the augmentation always works as a stabilization mechanism, see also the discussion in [5].

An important feature of the augmented Lagrangian approach is that it directly carries over to the case of inequality constraints, as first shown by Chouly and Hild in the context of elastic contact [16]. In our model problem we replace the constraint $u=g$ on $\Gamma$ by an inequality constraint $u-g \leq 0$ on $\Gamma$. We then have the following Kuhn-Tucker conditions on the multiplier and side condition:

$$
u-g \leq 0, \quad \lambda \leq 0, \quad \lambda(u-g)=0 .
$$

The key to incorporating these conditions into the augmented Lagrangian scheme, as pioneered by Alart and Curnier [1], is to make the observation that 9 is equivalent to

$$
\lambda=-\gamma\left[u-g-\gamma^{-1} \lambda\right]_{+} \text {and } u-g=\left[u-g-\gamma^{-1} \lambda\right]_{-}
$$

where $\gamma \in \mathbb{R}^{+},[x]_{ \pm}= \pm \max ( \pm x, 0)$, cf. [16]. Denoting $P_{\gamma}(u, \lambda):=\gamma(u-g)-\lambda$ we see that

$$
\lambda=-\left[P_{\gamma}(u, \lambda)\right]_{+}=\left[P_{\gamma}(u, \lambda)\right]_{-}-P_{\gamma}(u, \lambda) .
$$

We then formally write the augmented Lagrangian, similar to (8), but using the second relation in (10) for the contact constraint,

$$
\begin{aligned}
\mathscr{L}(v, \mu):=\frac{1}{2} a(v, v)-\left\langle\mu, v-g-[v-g-\gamma \mu]_{-}\right\rangle_{\Gamma} & \\
& +\frac{1}{2} \gamma\left\|v-g-\left[v-g-\gamma^{-1} \lambda\right]_{-}\right\|_{\Gamma}^{2}-(f, v)_{\Omega} .
\end{aligned}
$$

Observe that this naive formulation is not differentiable, so of little practical use. Our aim is now to propose a modified Lagrangian that is consistent with (12), but smoother. We use the relations 9 for the modifications, in order not to perturb the stationary points. The modifications must also respect the saddle point structure of the system. First observe that by the two relations of (10) the stationary point must satisfy $\left\langle\mu,[v-g-\gamma \mu]_{-}\right\rangle_{\Gamma}=0$, using this in the second term in the right hand side of (12) and developing the square of the third term we obtain

$$
\begin{aligned}
\mathscr{L}(v, \mu):=\frac{1}{2} a(v, v)-\langle\mu, v-g\rangle_{\Gamma}+\frac{1}{2} \gamma \| & v-g \|^{2} \\
& +\frac{1}{2} \gamma^{-1}\left\|\left[P_{\gamma}(u, \mu)\right]_{-}\right\|_{\Gamma}^{2}-\left\langle(v-g),\left[P_{\gamma}(u, \mu)\right]_{-}\right\rangle_{\Gamma}-(f, v)_{\Omega} .
\end{aligned}
$$

We then use that the stationary point must satisfy the second relation of 10$]$ implying that

$$
\left\langle(v-g),\left[P_{\gamma}(u, \mu)\right]_{-}\right\rangle_{\Gamma}=\gamma^{-1}\left\|\left[P_{\gamma}(u, \mu)\right]_{-}\right\|_{\Gamma}^{2}
$$

resulting in

$$
\mathscr{L}(v, \mu):=\frac{1}{2} a(v, v)-\langle\mu, v-g\rangle_{\Gamma}+\frac{1}{2} \gamma\|v-g\|^{2}
$$$$
-\frac{1}{2} \gamma^{-1}\left\|\left[P_{\gamma}(u, \mu)\right]_{-}\right\|_{\Gamma}^{2}-(f, v)_{\Omega} .
$$ 
This formulation is differentiable and the corresponding Euler-Lagrange equations read

$$
\begin{aligned}
a(u, v)-\langle\lambda, v\rangle_{\Gamma}+\langle\gamma u, v\rangle_{\Gamma}-\langle\mu, u\rangle_{\Gamma} & \\
& -\gamma^{-1}\left\langle\left[\gamma(u-g)-\partial_{n} u\right]_{-}, \gamma v-\mu\right\rangle_{\Gamma}=(f, v)_{\Omega}+\langle\gamma g, v\rangle_{\Gamma}-\langle\mu, g\rangle_{\Gamma} .
\end{aligned}
$$

Choosing now in the discrete case $\lambda_{h}:=\partial_{n} u_{h}$ and $\mu=\partial_{n} v$ results in a formulation that equals (7) up to a nonlinear perturbation: we seek $u_{h} \in V_{h}$ such that

$$
\begin{aligned}
a\left(u_{h}, v\right)-\left\langle\partial_{n} u_{h}, v\right\rangle_{\Gamma}-\left\langle\partial_{n} v_{h}, u_{h}\right\rangle_{\Gamma}+ & \left\langle\gamma u_{h}, v\right\rangle_{\Gamma} \\
& -\gamma^{-1}\left\langle\left[\gamma\left(u_{h}-g\right)-\partial_{n} u_{h}\right]_{-}, \gamma v-\partial_{n} v\right\rangle_{\Gamma}=L(v) \quad \forall v \in V_{h},
\end{aligned}
$$

where

for all $v \in V_{h}$.

$$
L(v):=(f, v)-\left\langle\partial_{n} v, g\right\rangle_{\Gamma}+\gamma\langle v, g\rangle_{\Gamma}
$$

To see the equivalence of this formulation with that introduced by Chouly and Hild [16] we once again use the relation (11) to write

$$
\begin{aligned}
& -\gamma^{-1}\left\langle\left[\gamma\left(u_{h}-g\right)-\partial_{n} u_{h}\right]_{-}, \gamma v-\partial_{n} v\right\rangle_{\Gamma}= \\
& -\left\langle\left[P_{\gamma}\left(u, \partial_{n} u\right)\right]_{-}-P_{\gamma}\left(u, \partial_{n} u\right), v-\gamma^{-1} \partial_{n} v\right\rangle_{\Gamma}-\left\langle P_{\gamma}\left(u, \partial_{n} u\right), v-\gamma^{-1} \partial_{n} v\right\rangle_{\Gamma} \\
& \quad=\left\langle\left[P_{\gamma}\left(u, \partial_{n} u\right)\right]_{+}, v-\gamma^{-1} \partial_{n} v\right\rangle_{\Gamma}-\left\langle P_{\gamma}\left(u, \partial_{n} u\right), v-\gamma^{-1} \partial_{n} v\right\rangle_{\Gamma} .
\end{aligned}
$$

Applying this relation in the last term of the left hand side of (13) and simplifying results in we seek $u_{h} \in V_{h}$ such that

$$
a\left(u_{h}, v\right)+\left\langle\gamma\left[u_{h}-g-\gamma^{-1} \partial_{n} u_{h}\right]_{+}, v-\gamma^{-1} \partial_{n} v\right\rangle_{\Gamma}-\left\langle\gamma^{-1} \partial_{n} u_{h}, \partial_{n} v\right\rangle_{\Gamma}=(f, v),
$$

for all $v \in V_{h}$. With the choice $\gamma=\gamma_{0} / h$ (14) precisely coincides with the nonlinear Nitsche method proposed by Chouly and Hild [16]. This variational problem corresponds to stationarity of the Lagrangian

$$
\mathscr{L}(v, \mu):=\frac{1}{2} a(v, v)+\frac{1}{2}\left\|\gamma^{1 / 2}\left[v-g-\gamma^{-1} \mu\right]_{+}\right\|_{\Gamma}^{2}-\left\langle\gamma^{-1} \mu, \mu\right\rangle_{\Gamma}-(f, v)_{\Omega},
$$

cf. [1].

In the following we shall explore how the augmented Lagrangian approach can be used in different situations.

\section{Poisson's equation with general boundary conditions}

We first consider the simple case of imposing general boundary conditions in a finite element method with the mesh fitted to the boundary. Following [24] we consider the model problem

$$
\begin{aligned}
-\nabla \cdot(\varepsilon \nabla u) & =f \text { in } \Omega \\
\varepsilon \partial_{n} u & =\kappa^{-1}\left(u_{0}-u\right)+g \text { on } \Gamma=\partial \Omega,
\end{aligned}
$$

where $\varepsilon, u_{0}$, and $\kappa$ are given functions of sufficient regularity. The problem can be equivalently formulated as the stationary points of the Lagrangian

$$
\mathscr{L}(v, \mu):=\frac{1}{2} a(v, v)-\langle\mu, v\rangle_{\Gamma}-\frac{1}{2} \kappa\left\|\mu^{2}\right\|_{\Gamma}-(f, v)_{\Omega}+\left\langle\left(u_{0}+\kappa g\right), \mu\right\rangle_{\Gamma} .
$$

where

$$
a(u, v):=(\varepsilon \nabla u, \nabla v)_{\Omega} .
$$

The augmented Lagrangian is obtained by adding a scaled least squares term on the constraint. This leads to

$$
\begin{aligned}
\mathscr{L}(v, \mu):= & \frac{1}{2} a(v, v)-\langle\mu, v\rangle_{\Gamma}-\frac{1}{2} \kappa\|\mu\|_{\Gamma}^{2} \\
& +\frac{1}{2 \kappa}\left\|\kappa \mu+v-u_{0}-\kappa g\right\|_{\Gamma}^{2}-(f, v)_{\Omega}+\left\langle\left(u_{0}+\kappa g\right), \mu\right\rangle_{\Gamma} .
\end{aligned}
$$


Developing the square of the least squares contribution leads to the elimination of the multiplier and the form

$$
\mathscr{L}(v):=a(v, v)-\frac{1}{2 \kappa}\|v\|_{\Gamma}^{2}-(f, v)_{\Omega}-\left\langle\left(\kappa^{-1} u_{0}+g\right), v\right\rangle_{\Gamma} .
$$

The corresponding optimality system is the standard weak formulation of the Robin problem: find $u \in$ $H^{1}(\Omega)$ such that

$$
a(u, v)+\kappa^{-1}\langle u, v\rangle_{\Gamma}=(f, v)_{\Omega}+\left\langle\left(\kappa^{-1} u_{0}+g\right), v\right\rangle_{\Gamma}, \quad \forall v \in H^{1}(\Omega) .
$$

Restricting the formulation to finite dimensional spaces results in the finite element formulation: find $u_{h} \in V_{h}$ such that

$$
a\left(u_{h}, v_{h}\right)+\kappa^{-1}\left\langle u_{h}, v_{h}\right\rangle_{\Gamma}=\left(f, v_{h}\right)_{\Omega}+\left\langle\left(\kappa^{-1} u_{0}+g\right), v_{h}\right\rangle_{\Gamma} .
$$

We see that as the "compliance" $\kappa \rightarrow 0,\left.u\right|_{\Gamma} \rightarrow u_{0}$, implying a Dirichlet condition in the limit and as $\kappa \rightarrow \infty$ we recover the weak formulation for the Neumann problem with $\left.\varepsilon \partial_{n} u\right|_{\Gamma}=g$. As was pointed out in [24] the corresponding linear system becomes ill-posed if $\kappa<<h$. As a remedy for this we will temper the coefficient in front of the least squares term in the augmented Lagrangian. Indeed if we introduce

$$
S_{h}=\left(\kappa+h / \gamma_{\kappa}\right)^{-1},
$$

where $\gamma_{\kappa}$ is a free parameter, and use this as coefficient, we obtain the augmented Lagrangian

$$
\begin{aligned}
\mathscr{L}(v, \mu):= & \frac{1}{2} a(v, v)-\langle\mu, v\rangle_{\Gamma}-\frac{1}{2} \kappa\|\mu\|_{\Gamma}^{2} \\
& +\frac{S_{h}}{2}\left\|\kappa \mu+v-u_{0}-\kappa g\right\|_{\Gamma}^{2}-(f, v)_{\Omega}-\left\langle\left(u_{0}+\kappa g\right), \mu\right\rangle_{\Gamma} .
\end{aligned}
$$

The cancellations that led to the previous simple optimality system will not take place this time as the moderated parameter $S_{h}$ will prevent the system from becoming too stiff. Deriving we find the following optimality system

$$
\begin{aligned}
a(u, v)-\left\langle\left(1-\kappa S_{h}\right) \lambda, v\right\rangle_{\Gamma}-\left\langle\left(1-\kappa S_{h}\right) \mu, u\right\rangle_{\Gamma} & \\
& -\left\langle\kappa\left(1-S_{h} \kappa\right) \lambda, \mu\right\rangle_{\Gamma}+\left\langle S_{h} u, v\right\rangle_{\Gamma}= \\
& (f, v)_{\Omega}+\left\langle\left(u_{0}+\kappa g\right),\left(1-S_{h} \kappa\right) \mu+S_{h} v\right\rangle_{\Gamma} .
\end{aligned}
$$

Using this formulation in a finite element method with a pair $V_{h} \times \Lambda_{h}$ that satisfies the inf-sup condition leads to a robust and accurate method. Formally replacing $\lambda$ by $\varepsilon \partial_{n} u$ and $\mu$ by $\varepsilon \partial_{n} v$ and writing the form on the finite space $V_{h}$ yields the method: find $u_{h} \in V_{h}$ such that

$$
\begin{gathered}
a\left(u_{h}, v_{h}\right)-\left\langle\left(1-\kappa S_{h}\right) \varepsilon \partial_{n} u_{h}, v_{h}\right\rangle_{\Gamma}-\left\langle\left(1-\kappa S_{h}\right) \varepsilon \partial_{n} v_{h}, u_{h}\right\rangle_{\Gamma} \\
-\left\langle\kappa\left(1-S_{h} \kappa\right) \varepsilon \partial_{h} u_{h}, \varepsilon \partial_{h} v_{h}\right\rangle_{\Gamma}+\left\langle S_{h} u_{h}, v_{h}\right\rangle_{\Gamma} \\
=\left(f, v_{h}\right)_{\Omega}+\left\langle\left(u_{0}+\kappa g\right),\left(1-S_{h} \kappa\right) \varepsilon \partial_{h} v_{h}+S_{h} v_{h}\right\rangle_{\Gamma} .
\end{gathered}
$$

We identify the Nitsche method proposed in [24] and conclude that their analysis is valid for $u_{h}$ if the parameter $\gamma_{\kappa}$ is chosen large enough. Observe that the Lagrange multiplier formulation (22), which appears to be new, also varies robustly between a Neumann and a Dirichlet condition in the two limits, without succumbing to the ill-conditioning in the limits of high or low $\kappa$. We note in passing that sometimes the multiplier method can have advantages compared to Nitsche's method, in particular if fields defined on different meshes must be coupled, see for instance [10].

\section{FEM for Poisson's problem with discontinuous coefficients}

We are interested in the following problem: find $u_{i}: \Omega_{i} \mapsto \mathbb{R}, i=1,2$, such that

$$
\begin{aligned}
-\nabla \cdot \varepsilon_{i} \nabla u_{i} & =f \text { in } \Omega_{i}, i=1,2 \\
u_{i} & =0 \text { on } \partial \Omega \cap \Omega_{i} i=1,2 \\
{\left[\left[\varepsilon \partial_{n} u\right]\right] } & =g \text { on } \Gamma:=\partial \bar{\Omega}_{1} \cap \partial \bar{\Omega}_{2} \\
{[u]] } & =0 \text { on } \Gamma .
\end{aligned}
$$


We use the notation $u=\left(u_{1}, u_{2}\right) \in V_{1} \times V_{2}$ with the continuous spaces

$$
V_{i}=\left\{v_{i} \in H^{1}\left(\Omega_{i}\right): \partial v_{i} / \partial n_{i} \in L_{2}(\Gamma),\left.v_{i}\right|_{\partial \Omega \cap \partial \Omega_{i}}=0\right\}, \quad i=1,2 .
$$

Then $[[u]]$ denotes the jump of $u$ over $\Gamma$ defined as

$$
[[u]]:=\lim _{\varepsilon \rightarrow 0^{+}} u(x-\varepsilon n)-u(x+\varepsilon n)
$$

for $x \in \Gamma$ and $n$ denoting the normal on $\Gamma$ pointing from $\Omega_{1}$ to $\Omega_{2}$. The diffusion coefficients $\varepsilon_{i}, i=1,2$, are assumed to be constant functions. We will also use the weighted averages

$$
\left\{\{u\}_{w}:=\lim _{\varepsilon \rightarrow 0^{+}}\left(w_{1} u(x-\varepsilon n)+w_{2} u(x+\varepsilon n)\right)\right.
$$

and

$$
\left\langle\langle u\rangle_{w}:=\lim _{\varepsilon \rightarrow 0^{+}}\left(w_{2} u(x-\varepsilon n)+w_{1} u(x+\varepsilon n)\right)\right.
$$

where $w_{1}, w_{2} \geq 0$ are positive weights such that $w_{1}+w_{2}=0$. This problem can be shown to be equivalent to finding $(u, \lambda) \in H^{1}\left(\Omega_{1} \cup \Omega_{2}\right) \times H^{-\frac{1}{2}}(\Gamma)$, the saddle point of the constrained minimization problem defined by the Lagrangian

$$
\mathscr{L}(v, \mu):=\frac{1}{2} a(v, v)+\langle\mu,[[v]]\rangle_{-\frac{1}{2}, \frac{1}{2}, \Gamma}-(f, v)_{\Omega}-\left\langle g,\langle\langle v\rangle\rangle_{w}\right\rangle_{\Gamma} .
$$

where now

Thus, $(u, \lambda)$ fulfills

$$
a(u, v):=(\varepsilon \nabla u, \nabla v)_{\Omega_{1} \cup \Omega_{2}}
$$

$$
\mathscr{L}(u, \lambda)=\inf _{v \in V} \sup _{\mu \in \Lambda} \mathscr{L}(v, \mu)
$$

We also know that for the exact solution there holds $\lambda=-\left\{\{\varepsilon \nabla u \cdot n\}_{w}\right.$, for any admissible weights $w_{1}, w_{2}$.

To formulate a discrete method, we suppose that we have regular finite element partitionings $\mathscr{T}_{h}^{i}$ of the subdomains $\Omega_{i}$ into shape regular simplexes. These two meshes have corresponding trace meshes on the interface and for simplicity we assume that the meshes match across the interface so that the trace meshes are equivalent and we may write

$$
\mathscr{F}_{h}=\left\{F: F=T \cap \Gamma, T \in \mathscr{T}_{h}^{i}, i=1 \text { or } 2\right\} .
$$

We seek the approximation $u_{h}=\left(u_{1, h}, u_{2, h}\right)$ in the space $V^{h}=V_{1}^{h} \times V_{2}^{h}$, where

$$
V_{i}^{h}=\left\{v_{i} \in V_{i}:\left.v_{i}\right|_{T} \in \mathbb{P}_{k}(T), \forall T \in \mathscr{T}_{h}\right\}, \quad \text { for } k \geq 1,
$$

and for $\lambda_{h}$ in

$$
\Lambda_{h}:=\left\{q_{h} \in L_{2}(\Gamma):\left.q_{h}\right|_{F} \in \mathbb{P}_{l}(F), \forall F \in \mathscr{F}_{h}\right\}, \quad \text { for } l \geq 0 .
$$

If we now restrict the infimum of the supremum in 28 to our finite dimensional subspaces $V_{h}$ and $\Lambda_{h}$ chosen such that the discrete spaces satisfy the inf-sup condition, we immediately obtain the standard Lagrange multiplier domain decomposition method [4], with the Lagrangian given by

$$
\mathscr{L}\left(v_{h}, \mu_{h}\right):=\frac{1}{2} a\left(v_{h}, v_{h}\right)+\left\langle\mu_{h},\left[\left[v_{h}\right]\right]\right\rangle_{\Gamma}-\left(f, v_{h}\right)_{\Omega}-\left\langle g,\left\langle\left\langle v_{h}\right\rangle\right\rangle_{w}\right\rangle_{\Gamma} .
$$

The augmented Lagrangian is obtained by adding a least squares penalty on the constraint:

$$
\left.\mathscr{L}\left(v_{h}, \mu_{h}\right):=\frac{1}{2} a\left(v_{h}, v_{h}\right)+\left\langle\mu_{h},\left[\left[v_{h}\right]\right]\right\rangle_{\Gamma}+\frac{\gamma}{2} \|\left[v_{h}\right]\right] \|_{\Gamma}^{2}-\left(f, v_{h}\right)_{\Omega}-\left\langle g,\left\langle\left\langle v_{h}\right\rangle\right\rangle_{w}\right\rangle_{\Gamma} .
$$

This allows us to instead look for stationary points of the following augmented Lagrangian since we are working in discrete spaces and all pairings are $L^{2}$ scalar products:

$$
\begin{aligned}
\mathscr{L}\left(v_{h}, \mu_{h}\right):= & \left.\frac{1}{2} a\left(v_{h}, v_{h}\right)+\frac{1}{2 \gamma} \| \mu_{h}+\gamma\left[v_{h}\right]\right] \|_{\Gamma}^{2} \\
& -\frac{1}{2}\left\langle\gamma^{-1} \mu_{h}, \mu_{h}\right\rangle_{\Gamma}-\left(f, v_{h}\right)_{\Omega}-\left\langle g,\left\langle\left\langle v_{h}\right\rangle_{w}\right\rangle_{\Gamma} .\right.
\end{aligned}
$$


The Euler-Lagrange equations characterising the saddle point of the system (if it exists) takes the form: find $\left(u_{h}, \lambda_{h}\right) \in V_{h} \times \Lambda_{h}$ such that

$$
\begin{aligned}
a\left(u_{h}, v_{h}\right)+\gamma^{-1}\left\langle\lambda_{h}+\gamma\left[\left[u_{h}\right]\right], \mu_{h}+\gamma\left[\left[v_{h}\right]\right]\right\rangle_{\Gamma}-\left\langle\gamma^{-1} \lambda_{h}, \mu_{h}\right\rangle_{\Gamma}= & \left(f, v_{h}\right)_{\Omega} \\
& +\left\langle g,\left\langle\left\langle v_{h}\right\rangle\right\rangle_{w}\right\rangle_{\Gamma}
\end{aligned}
$$

for all $v_{h}, \mu_{h} \in V_{h} \times \lambda_{h}$. Developing the second term of the left hand side we see that this is equivalent to

$$
\begin{aligned}
a\left(u_{h}, v_{h}\right)+\left\langle\lambda_{h}+\gamma\left[\left[u_{h}\right]\right],\left[\left[v_{h}\right]\right]\right\rangle_{\Gamma} & =\left(f, v_{h}\right)_{\Omega}+\left\langle g,\left\langle\left\langle v_{h}\right\rangle\right\rangle_{w}\right\rangle_{\Gamma} \\
\left\langle\left[\left[u_{h}\right]\right], \mu_{h}\right\rangle_{\Gamma} & =0 .
\end{aligned}
$$

This shows that the effect of the augmented Lagrangian compared to the standard Lagrange multiplier method is simply the addition of a penalty term on the constraint which, as mentioned above, gives us stronger control of the constraints than would otherwise be possible. Indeed for the standard Lagrange multiplier method only $\pi_{\Lambda}\left[\left[u_{h}\right]\right]$ is controlled, where $\left\langle\pi_{\Lambda}\left[\left[u_{h}\right]\right], \mu_{h}\right\rangle_{\Gamma}=\left\langle\left[\left[u_{h}\right]\right], \mu_{h}\right\rangle_{\Gamma}$ for all $\mu_{h} \in \Lambda_{h}$. This formulation is however stable only for well balanced choices of $V_{h}$ and $\Lambda_{h}$. In case the spaces do not satisfy the inf-sup condition one may add a stabilizing term $j\left(\lambda_{h}, \lambda_{h}\right)$ satisfying

$$
\left\|h^{\frac{1}{2}}\left(\lambda_{h}-\pi_{\Gamma} \lambda_{h}\right)\right\|_{\Gamma} \lesssim j\left(\lambda_{h}, \lambda_{h}\right)^{\frac{1}{2}}
$$

where $\pi_{\Gamma}$ satisfies $\left\langle\pi_{\Gamma} \mu_{h}, v_{h}\right\rangle_{\Gamma}=\left\langle\mu_{h}, v_{h}\right\rangle_{\Gamma}$ for all $v_{h}$ in the trace mesh of $\mathscr{T}_{1}$ (or $\mathscr{T}_{2}$ ). For instance if $V_{h}$ is the space of piecewise affine, continuous functions in each subdomain and $\Lambda_{h}$ is the space of piecewise constant functions defined on the elements cut by $\Gamma$ we may choose

$$
j\left(\lambda_{h}, \lambda_{h}\right):=\sum_{F \in \mathscr{F}}\left\|h\left[\left[\lambda_{h}\right]\right]\right\|_{\partial F \backslash \partial \Gamma}^{2}
$$

and follow the analysis of [10] to prove error estimates for the formulation: find $\left(u_{h}, \lambda_{h}\right) \in V_{h} \times \Lambda_{h}$ such that

$$
\begin{aligned}
a\left(u_{h}, v_{h}\right)+\left\langle\lambda_{h}+\gamma\left[\left[u_{h}\right]\right],\left[\left[v_{h}\right]\right]\right\rangle_{\Gamma} & =\left(f, v_{h}\right)_{\Omega}+\left\langle g,\left\langle\left\langle v_{h}\right\rangle\right\rangle_{w}\right\rangle_{\Gamma} \\
\left\langle\left[\left[u_{h}\right]\right], \mu_{h}\right\rangle_{\Gamma}-j\left(\lambda_{h}, \mu_{h}\right) & =0
\end{aligned}
$$

for all $v_{h}, \mu_{h} \in V_{h} \times \lambda_{h}$. On the other hand, if we formally replace $\lambda_{h}$ by $-\left\{\left\{\mu \partial_{n} u_{h}\right\}_{w}\right.$ and $\mu_{h}$ by $-\left\{\left\{\mu \partial_{h} v_{h}\right\}_{w}\right.$ we obtain the formulation: find $u_{h} \in V_{h}$ such that

$$
\begin{array}{r}
a\left(u_{h}, v_{h}\right)-\left\langle\left\{\left\{\mu \partial_{n} u_{h}\right\}_{w},\left[\left[v_{h}\right]\right]\right\rangle_{\Gamma}-\left\langle\left[\left[u_{h}\right]\right],\left\{\left\{\mu \partial_{n} v_{h}\right\}\right\}_{w}\right\rangle_{\Gamma}+\left\langle\gamma\left[\left[u_{h}\right]\right],\left[\left[v_{h}\right]\right]\right\rangle_{\Gamma}\right. \\
=\left(f, v_{h}\right)_{\Omega}+\left\langle g,\left\langle\left\langle v_{h}\right\rangle\right\rangle_{w}\right\rangle_{\Gamma}
\end{array}
$$

for all $v_{h} \in V_{h}$, and we recognise Nitsche's formulation from [21].

\section{Debonding and adhesive contact}

A robust discretization of the debonding problem was proposed in [22]. We will revisit their arguments in the context of Lagrange multipliers as an augmented Lagrangian formulation. The linear model problem in this case takes the form

$$
\begin{aligned}
-\nabla \cdot \varepsilon \nabla u & =f \text { in } \Omega_{1} \cup \Omega_{2} \\
u & =0 \text { on } \partial \Omega \\
{\left.\left[\varepsilon \partial_{n} u\right]\right] } & =0 \text { on } \Gamma \\
{[[u]] } & =-\kappa\left\{\left\{\varepsilon \partial_{n} u\right\}_{w} \text { on } \Gamma .\right.
\end{aligned}
$$

Here we note that by the continuity of the fluxes the formulation is independent of the choice of the weights $w_{i}, i=1,2$. This time the physical solution is discontinuous over the boundary and $u \in H^{1}\left(\Omega_{1} \cup\right.$ $\left.\Omega_{2}\right)$.

The critical case is when $\kappa$ becomes large and a naive coupling strategy leads to an ill-conditioned system or even locking on the interface. We therefore follow [22], but contrary to the discussion in that paper we herein use the augmented Lagrangian formulation to arrive at the method. In the intermediate step we obtain a robust Lagrange multiplier method for the debonding problem. This time we start 
from the following Lagrangian, the saddle points of which coincides with the solution of the debonding problem,

$$
\mathscr{L}(v, \mu):=\frac{1}{2} a(v, v)-\langle\mu,[[v]]\rangle_{-\frac{1}{2}, \frac{1}{2}, \Gamma}-\frac{1}{2} \kappa\|\mu\|_{\Gamma}^{2}-(f, v)_{\Omega} .
$$

The augmented Lagrangian is obtained adding a least squares term on the constraint

$$
\left.\mathscr{L}(v, \mu):=\frac{1}{2} a(v, v)-\langle\mu,[[v]]\rangle_{-\frac{1}{2}, \frac{1}{2}, \Gamma}-\frac{1}{2} \kappa\|\mu\|_{\Gamma}^{2}+\frac{1}{2 \kappa} \|[v]\right]+\kappa \mu \|_{\Gamma}^{2}-(f, v)_{\Omega} .
$$

Developing the square we see that the multiplier is eliminated and we obtain

$$
\mathscr{L}(v):=\frac{1}{2} a(v, v)+\frac{1}{2 \kappa} \|\left[[v] \|_{\Gamma}^{2}-(f, v)_{\Omega} .\right.
$$

Studying the corresponding optimality system leads to: find $u_{h} \in V_{h}$ such that

$$
a\left(u_{h}, v_{h}\right)+S\left\langle\left[\left[u_{h}\right]\right],[[v]]\right\rangle_{\Gamma}=(f, v)_{\Omega}, \quad \forall v_{h} \in V_{h} .
$$

where $S=\kappa^{-1}$. As for the problem in Section 3 this formulation becomes ill-conditioned for $\kappa$ small since the two terms of the left hand side will have sizes of different orders of magnitude. A possible remedy is to replace $\kappa$ by $\max (h, \kappa)$ in equation 41 , but this results in a nonconsistent perturbation of the system and reduced accuracy, in the regime where $\kappa<h$.

If we instead modify the size of the least squares contribution in the augmented Lagrangian we can moderate the strength of the imposition of the constraint in a consistent manner. Introducing the parameter

$$
S_{h}=\left(h_{k} / \gamma_{\kappa}+\kappa\right)^{-1},
$$

similarly as for the method in Section 3 , we may write

$$
\left.\mathscr{L}(v, \mu):=\frac{1}{2}\left\|\varepsilon^{\frac{1}{2}} \nabla v\right\|_{\Omega}^{2}-\langle\mu,[[v]]\rangle_{-\frac{1}{2}, \frac{1}{2}, \Gamma}-\frac{1}{2} \kappa\|\mu\|_{\Gamma}^{2}+\frac{1}{2} S_{h} \|[v]\right]+\kappa \mu \|_{\Gamma}^{2}-(f, v)_{\Omega} .
$$

Observe that the saddle point to this system is a weak solution to 36-39, but the size of the weight in front of the least squares term can never be larger than $\gamma_{\kappa} / h$, effectively bounding the stiffness of the system. The corresponding optimality system now reads:

$$
\begin{aligned}
a(u, v)-\left\langle\left(1-\kappa S_{h}\right) \lambda,[[v]]\right\rangle_{\Gamma}-\left\langle\left(1-\kappa S_{h}\right) \mu,[[u]]\right\rangle_{\Gamma} & \\
& -\left\langle\kappa\left(1-S_{h} \kappa\right) \lambda, \mu\right\rangle_{\Gamma}+\left\langle S_{h}[[u],[[v]]\rangle_{\Gamma}=(f, v)_{\Omega} .\right.
\end{aligned}
$$

Formally replacing $\lambda$ by $\left\{\left\{\varepsilon \partial_{n} u\right\}_{w}\right.$ and $\mu$ by $\left\{\left\{\varepsilon \partial_{n} v\right\}_{w}\right.$ and writing the form on the finite space $V_{h}$ yields: find $u_{h} \in V_{h}$ such that

$$
\begin{aligned}
a\left(u_{h}, v_{h}\right)-\left\langle\left(1-\kappa S_{h}\right)\left\{\left\{\varepsilon \partial_{h} u_{h}\right\}_{w},\left[\left[v_{h}\right]\right]\right\rangle_{\Gamma}-\left\langle\left(1-\kappa S_{h}\right)\left\{\left\{\varepsilon \partial_{h} v_{h}\right\}_{w},\left[\left[u_{h}\right]\right]\right\rangle_{\Gamma}\right.\right. \\
-\left\langle\kappa\left(1-S_{h} \kappa\right)\left\{\left\{\varepsilon \partial_{h} u_{h}\right\}\right\}_{w},\left\{\left\{\varepsilon \partial_{h} v_{h}\right\}_{w}\right\rangle_{\Gamma}\right. \\
+\left\langle S_{h}\left[\left[u_{h}\right]\right],\left[\left[v_{h}\right]\right]\right\rangle_{\Gamma}=\left(f, v_{h}\right)_{\Omega} .
\end{aligned}
$$

We recognise the same Nitsche type method as proposed in [22], but this time with the weights chosen as in the previous section to also be robust with respect to the contrast in the diffusivity.

\subsection{Adhesive contact}

In the previous linear model only the adhesive forces are accounted for, which implies that penetration is possible. A more physically realistic model excludes penetration by formulating the problem as a variational inequality. Our last model problem concerns this nonlinear model and we will combine the arguments developed above with those of [16 17]. Here for simplicity we assume that both $\Omega_{1}$ and $\Omega_{2}$ intersects the boundary $\partial \Omega$.

$$
\begin{aligned}
-\nabla \cdot \varepsilon \nabla u & =f \text { in } \Omega_{1} \cup \Omega_{2} \\
u & =0 \text { on } \partial \Omega \\
{\left.\left[\varepsilon \varepsilon \partial_{n} u\right]\right] } & =0 \text { on } \Gamma \\
{[[u]] } & \leq 0 \text { on } \Gamma \\
\kappa^{-1}[[u]]+\left\{\left\{\varepsilon \partial_{n} u\right\}_{w}\right. & \leq 0 \text { on } \Gamma \\
{[[u]]\left(\kappa^{-1}[[u]]+\left\{\left\{\varepsilon \partial_{n} u\right\}_{w}\right)\right.} & =0 \text { on } \Gamma
\end{aligned}
$$


To cast this problem on the form of an augmented Lagrangian method we start out with the functional 40] with an additional constraint on $[[v]]$ :

$$
\mathscr{L}(v, \mu):=\frac{1}{2}\left\|\varepsilon^{\frac{1}{2}} \nabla v\right\|_{\Omega}^{2}-\langle\mu,[[v]]\rangle_{-\frac{1}{2}, \frac{1}{2}, \Gamma}+\frac{1}{2 \kappa}\|[[v]]\|_{\Gamma}^{2}-(f, v)_{\Omega} .
$$

The Euler-Lagrange equations are to find $(u, \lambda)$ such that

$$
\begin{gathered}
a(u, v)-\langle\lambda,[[v]]\rangle_{-\frac{1}{2}, \frac{1}{2}, \Gamma}+\frac{1}{\kappa}\langle[[u]],[[v]]\rangle_{\Gamma}=(f, v)_{\Omega} \quad \forall v \in H^{1}\left(\Omega_{1} \cup \Omega_{2}\right), \\
\left\langle\mu,[[u]\rangle_{-\frac{1}{2}, \frac{1}{2}, \Gamma}=0 \quad \forall \mu \in H^{-\frac{1}{2}}(\Gamma),\right.
\end{gathered}
$$

and we note from 51 that, formally, the multiplier is given by

$$
\lambda=\left\{\left\{\varepsilon \partial_{n} u\right\}_{w}+\kappa^{-1}[[u]]\right.
$$

but of course $[52$ enforces $[[u]]=0$ weakly. In order to create a one-sided contact condition we now consider the Kuhn-Tucker conditions 47-449) as

$$
\lambda \leq 0,[[u]] \leq 0 \text {, and } \lambda[[u]]=0 \text { on } \Gamma .
$$

These conditions can equivalently be formulated as

$$
\left.\lambda=-\gamma[[u]]-\gamma^{-1} \lambda\right]_{+},
$$

where $[x]_{+}=\max (x, 0)$, cf. [16]. To introduce this condition in the Lagrangian [50] we note that, if $\lambda, \mu \in L^{2}(\Gamma)$ (or if $\gamma^{-1}: H^{-\frac{1}{2}}(\Gamma) \mapsto H^{\frac{1}{2}}(\Gamma)$, with suitable properties), in 51) we have

$$
-\langle\lambda,[[v]]\rangle_{\Gamma}=-\left\langle\lambda,[[v]]-\gamma^{-1} \mu\right\rangle_{\Gamma}-\left\langle\lambda, \gamma^{-1} \mu\right\rangle_{\Gamma} .
$$

Using now 55 in the first term of the right hand side we have

$$
\left.\left.a(u, v)+\left\langle\gamma[[u]]-\gamma^{-1} \lambda\right]_{+},[[v]]-\gamma^{-1} \mu\right)\right\rangle_{\Gamma}+\frac{1}{\kappa}\langle[[u]],[[v]]\rangle_{\Gamma}=(f, v)_{\Omega} .
$$

Moving over to discrete spaces, where the assumption on $\lambda_{h}$ makes sense, we write the corresponding Lagrangian on augmented form as

$$
\left.\left.\mathscr{L}_{\mathrm{a}}\left(v_{h}, \mu_{h}\right):=a\left(v_{h}, v_{h}\right)+\frac{\gamma}{2} \|\left[\left[v_{h}\right]\right]-\gamma^{-1} \mu_{h}\right]_{+}\left\|_{\Gamma}^{2}-\frac{1}{2 \gamma}\right\| \mu_{h}\left\|_{\Gamma}^{2}+\frac{1}{2 \kappa}\right\|\left[v_{h}\right]\right] \|_{\Gamma}^{2}-\left(f, v_{h}\right)_{\Omega}
$$

leading to the problem of finding $\left(u_{h}, \lambda_{h}\right) \in V_{h} \times \Lambda_{h}$ such that

$$
\begin{array}{rr}
\left.a\left(u_{h}, v_{h}\right)+\gamma\left\langle\left[\left[\left[u_{h}\right]\right]-\gamma^{-1} \lambda\right]_{+},\left[\left[v_{h}\right]\right]-\gamma^{-1} \mu_{h}\right)\right\rangle_{\Gamma}-\gamma^{-1}\left\langle\lambda_{h}, \mu_{h}\right\rangle_{\Gamma} & \\
+\frac{1}{\kappa}\left\langle\left[\left[u_{h}\right]\right],\left[\left[v_{h}\right]\right]\right\rangle_{\Gamma}=\left(f, v_{h}\right)_{\Omega}
\end{array}
$$

for all $\left(v_{h}, \mu_{h}\right) \in V_{h} \times \Lambda_{h}$.

Inserting now the definition (53) of the multiplier and choosing $\mu=\left\{\left\{\varepsilon \partial_{n} v\right\}_{w}+\kappa^{-1}[[v]]\right.$ we seek $u_{h} \in V_{h}$ such that

$$
\begin{aligned}
& a\left(u_{h}, v_{h}\right)+\frac{1}{\kappa}\left\langle\left[\left[u_{h}\right]\right],\left[\left[v_{h}\right]\right]\right\rangle_{\Gamma} \\
& +\gamma\left\langle\left[\left(1-(\gamma \kappa)^{-1}\right)\left[\left[u_{h}\right]\right]-\gamma^{-1}\left\{\left\{\varepsilon \partial_{h} u_{h}\right\}_{w}\right]_{+},\left(1-(\gamma \kappa)^{-1}\right)[[v]]-\gamma^{-1}\left\{\left\{\varepsilon \partial_{n} v\right\}_{w}\right)\right\rangle_{\Gamma}\right. \\
& -\gamma^{-1}\left\langle\left\{\left\{\varepsilon \partial_{n} u_{h}\right\}_{w}+\kappa^{-1}\left[\left[u_{h}\right]\right],\left\{\left\{\varepsilon \partial_{n} v_{h}\right\}_{w}+\kappa^{-1}\left[\left[v_{h}\right]\right]\right\rangle_{\Gamma}=\left(f, v_{h}\right)_{\Omega} \quad \forall v_{h} \in V_{h} .\right.\right.
\end{aligned}
$$

It is instructive to consider the two limiting cases of full contact and of no contact: at contact, [57] gives

$$
\begin{aligned}
& a\left(u_{h}, v_{h}\right)-\left\langle\left[\left[u_{h}\right]\right],\left\{\left\{\varepsilon \partial_{h} v_{h}\right\}_{w}\right\rangle_{\Gamma}-\left\langle\left[\left[v_{h}\right]\right],\left\{\left\{\varepsilon \partial_{h} u_{h}\right\}_{w}\right\rangle_{\Gamma}\right.\right. \\
& +\left(\gamma-\frac{1}{\kappa}\right)\left\langle\left[\left[u_{h}\right]\right],\left[\left[v_{h}\right]\right]\right\rangle_{\Gamma}=\left(f, v_{h}\right)_{\Omega} \quad \forall v_{h} \in V_{h} .
\end{aligned}
$$


With the particular choice

$$
\gamma:=\frac{\gamma_{\kappa}}{h}+\frac{1}{\kappa}
$$

we obtain the following discrete problem: find $u_{h} \in V_{h}$ such that

$$
a\left(u_{h}, v_{h}\right)-\left\langle\left\{\left\{\varepsilon \partial_{n} u_{h}\right\}_{w}\left[\left[v_{h}\right]\right]\right\rangle-\left\langle\left\{\left\{\varepsilon \partial_{n} v_{h}\right\}_{w}\left[\left[u_{h}\right]\right]\right\rangle+\frac{\gamma_{\kappa}}{h}\left\langle\left[\left[u_{h}\right]\right],\left[\left[v_{h}\right]\right]\right\rangle_{\Gamma}=\left(f, v_{h}\right)_{\Omega}\right.\right.
$$

for all $v_{h} \in V_{h}$, which is the standard Nitsche method (35) for the adhesion free problem (with $\gamma_{\kappa}=\gamma_{0}$ ). In the case of no contact observe that by using $\gamma^{-1}=\gamma^{-1}-\kappa+\kappa$,

$$
\begin{aligned}
& \frac{1}{\kappa}\left\langle\left[\left[u_{h}\right]\right],\left[\left[v_{h}\right]\right]\right\rangle_{\Gamma}-\gamma^{-1}\left\langle\left\{\left\{\varepsilon \partial_{n} u_{h}\right\}_{w}+\kappa^{-1}\left[\left[u_{h}\right]\right],\left\{\left\{\varepsilon \partial_{n} v_{h}\right\}\right\}_{w}+\kappa^{-1}\left[\left[v_{h}\right]\right]\right\rangle_{\Gamma}\right. \\
& =-\left\langle\left\{\left\{\varepsilon \partial_{n} u_{h}\right\}_{w},\left[\left[v_{h}\right]\right]+\kappa\left\{\left\{\varepsilon \partial_{n} v_{h}\right\}_{w}\right\rangle_{\Gamma}\right.\right. \\
& -\left\langle\left[\left[u_{h}\right]\right]+\kappa\left\{\left\{\varepsilon \partial_{n} u_{h}\right\}_{w},\left\{\left\{\varepsilon \partial_{n} v_{h}\right\}_{w}\right\rangle_{\Gamma}-\left\langle\kappa\left\{\left\{\varepsilon \partial_{n} u_{h}\right\}\right\}_{w},\left\{\left\{\varepsilon \partial_{n} v_{h}\right\}\right\}_{w}\right\rangle_{\Gamma}\right.\right. \\
& \left(\kappa-\gamma^{-1}\right)\left\langle\left\{\left\{\varepsilon \partial_{n} u_{h}\right\}_{w}+\kappa^{-1}\left[\left[u_{h}\right]\right],\left\{\left\{\varepsilon \partial_{n} v_{h}\right\}_{w}+\kappa^{-1}\left[\left[v_{h}\right]\right]\right\rangle_{\Gamma} .\right.\right.
\end{aligned}
$$

The finite element formulation then takes the form: we seek $u_{h} \in V_{h}$ such that

$$
\begin{aligned}
\left(f, v_{h}\right)_{\Omega}= & a\left(u_{h}, v_{h}\right)-\left\langle\left\{\left\{\varepsilon \partial_{n} u_{h}\right\}\right\}_{w},\left[\left[v_{h}\right]\right]+\kappa\left\{\left\{\varepsilon \partial_{n} v_{h}\right\}\right\}_{w}\right\rangle_{\Gamma} \\
& \left.-\left\langle\left[\left[u_{h}\right]\right]+\kappa\left\{\left\{\varepsilon \partial_{n} u_{h}\right\}\right\}_{w},\left\{\left\{\varepsilon \partial_{n} v_{h}\right\}\right\}_{w}\right\rangle_{\Gamma}-\left\langle\kappa\left\{\varepsilon \partial_{n} u_{h}\right\}\right\}_{w},\left\{\left\{\varepsilon \partial_{h} v_{h}\right\}\right\}_{w}\right\rangle_{\Gamma} \\
& +\frac{1}{\gamma_{1}}\left\langle\left[\left[u_{h}\right]\right]+\kappa\left\{\left\{\varepsilon \partial_{n} u_{h}\right\}_{w},\left[\left[v_{h}\right]\right]+\kappa\left\{\left\{\varepsilon \partial_{n} v_{h}\right\}_{w}\right\rangle_{\Gamma}, \quad \forall v_{h} \in V_{h},\right.\right.
\end{aligned}
$$

where

$$
\gamma_{1}:=\frac{\kappa^{2}}{\kappa-\gamma^{-1}}=\kappa+\frac{h}{\gamma_{\kappa}}
$$

which coincides with the form 43.

\section{Stabilization for the extension to CutFEM}

Contrary to the methods discussed above, where the domains are meshed in the usual way, the CutFEM approach instead represents the boundary of a given domain on a background grid, for instance using a level set function. The background grid is then also used to represent the approximate solution of the governing partial differential equations. Consequently, CutFEM eases the burden of mesh generation by requiring only a low-quality surface mesh representation of the computational geometry. Cutting the mesh will, however, result in boundary elements with very small intersection with the physical domain. This may lead to a poorly conditioned system matrix or failure of stability of the discrete scheme. A remedy to this problem is to add a penalty term in the cut element zone that extends the coercivity to the whole mesh domain, i.e., in the $O(h)$ zone of the mesh domain (of each subdomain for interface problems) that does not intersect the associated physical domain. This penalty term, termed ghost penalty due to it acting partly outside of the domain of interest, must be carefully designed to add sufficient stability, while remaining weakly consistent for smooth solutions. The basic methods described above are then applied to the cut meshes and the only additional term is the ghost penalization.

To illustrate this idea, we consider the CutFEM method for the Poisson problem (7). We observe that by taking $v=u_{h}$ in the bilinear form $a\left(u_{h}, v\right)$, we have the coercivity

$$
\left\|\nabla u_{h}\right\|_{L^{2}(\Omega)}^{2} \leq a\left(u_{h}, u_{h}\right) .
$$

However, to obtain coercivity of the form $a_{h}\left(u_{h}, v\right)$ using this stability and the boundary penalty term, the penalty parameter will depend on how the elements are cut, since, denoting the set of elements that are cut by $\Gamma$ by

$$
\mathscr{G}_{h}:=\left\{K \in \mathscr{T}_{h}: K \cap \Gamma \neq \emptyset\right\}
$$

we have, with

$$
a_{h}\left(u_{h}, v\right):=a\left(u_{h}, v\right)-\left\langle\partial_{n} u_{h}, v\right\rangle_{\Gamma}-\left\langle\partial_{n} v_{h}, u_{h}\right\rangle_{\Gamma}+\left\langle\gamma u_{h}, v\right\rangle_{\Gamma}
$$

that

$$
\begin{aligned}
a_{h}\left(u_{h}, u_{h}\right) \geq & \left\|\nabla u_{h}\right\|_{L^{2}(\Omega)}^{2}+\left\|\gamma^{\frac{1}{2}} u_{h}\right\|_{L^{2}(\Gamma)}^{2} \\
& -2 \sum_{K \in \mathscr{G}_{h}}\left\|\nabla u_{h}\right\|_{L^{2}(\Gamma \cap K)}\left\|u_{h}\right\|_{L^{2}(\Gamma \cap K)} .
\end{aligned}
$$


Using the following well known trace inequality: under reasonable mesh assumptions there exists a constant $C_{T}$, depending on $\Gamma$ but independent of the mesh, such that

$$
\|w\|_{L_{2}\left(\Gamma_{K}\right)}^{2} \leq C_{T}\left(h_{K}^{-1}\|w\|_{L_{2}(K)}^{2}+h_{K}\|\nabla w\|_{L_{2}(K)}^{2}\right), \quad \forall w \in H^{1}(K)
$$

we have,

$$
\left\|\nabla u_{h}\right\|_{L^{2}(\Gamma \cap K)} \leq C_{\Gamma}\left(\frac{|K \cap \Gamma|}{|K \cap \Omega|}\right)^{\frac{1}{2}}\left\|\nabla u_{h}\right\|_{L^{2}(K \cap \Omega)},
$$

where $|\cdot|$ denotes the measure of the indicated quantity. It follows that in principle we obtain coercivity by choosing

$$
\left.\gamma\right|_{K}>2 C_{\gamma}^{2}\left(\frac{|K \cap \Gamma|}{|K \cap \Omega|}\right),
$$

since by an arithmetic-geometric inequality, we have

$$
\begin{aligned}
a_{h}\left(u_{h}, u_{h}\right) \geq & \left\|\nabla u_{h}\right\|_{L^{2}(\Omega)}^{2}+\left\|\gamma^{\frac{1}{2}} u_{h}\right\|_{L^{2}(\Gamma)}^{2}-\frac{1}{2}\left\|\nabla u_{h}\right\|_{L^{2}(\Omega)}^{2} \\
& -\sum_{K \in \mathscr{G}_{h}} C_{\Gamma}^{2}\left(\frac{|K \cap \Gamma|}{|K \cap \Omega|}\right)\left\|u_{h}\right\|_{L^{2}(K \cap \Gamma)}^{2} \\
\geq & \frac{1}{2}\left\|\nabla u_{h}\right\|_{L^{2}(\Omega)}^{2}+\left\|\left(\gamma-2 C_{\Gamma}^{2}\left(\frac{|K \cap \Gamma|}{|K \cap \Omega|}\right)\right)^{\frac{1}{2}} u_{h}\right\|_{L^{2}(\Gamma)}^{2} .
\end{aligned}
$$

Unfortunately this makes $\gamma$ strongly dependent on the cut, since for $|K \cap \Gamma|=O\left(h_{K}\right)$, the volume measure $|K \cap \Omega|$ can be arbitrarily small, resulting in problems both with conditioning and accuracy. A solution to this problem is to add a stabilizing term $g_{h}\left(u_{h}, v\right)$ to the form $a_{h}(\cdot, \cdot)$. The role of this term is to extend the coercivity from the physical domain $\Omega$ to the mesh domain $\Omega_{\mathscr{T}}:=\Omega \cup \mathscr{G}_{h}$. In order to have this effect, the stabilization term should have the following properties.

1. It should give a bound on the energy norm on the mesh domain in the sense that

$$
c_{G}\left\|\nabla u_{h}\right\|_{\Omega_{\mathscr{T}}}^{2} \leq\left\|\nabla u_{h}\right\|_{\Omega}^{2}+g_{h}\left(u_{h}, u_{h}\right),
$$

where $c_{G}>0$ is bounded away from zero independent of the mesh/boundary intersection for positive ghost penalty stabilization parameter $\gamma_{g}$.

2. For an interpolant of the extension of $u, i_{h} u:=i_{h} \mathbb{E} u$ we must have the weak consistency

$$
g_{h}\left(i_{h} u, i_{h} u\right) \leq C h^{k}\|u\|_{H^{k+1}(\Omega)} .
$$

where the constant $C$ is independent of the mesh/boundary intersection.

One example of such a term is the ghost penalty stabilization

$$
g_{h}\left(u_{h}, v\right):=\sum_{F \in \mathscr{F}_{G}}\left(\gamma_{g} h\left[\left[\partial_{n_{F}} u_{h}\right]\right],\left[\left[\partial_{n_{F}} v\right]\right]\right)_{F},
$$

valid for piecewise affine approximation. Here, we introduced the set of element faces $\mathscr{F}_{G}$ associated with $\mathscr{G}_{h}$, defined as follows: for each face $F \in \mathscr{F}_{G}$ there exists two simplices $K$ and $K^{\prime}$ such that $F=$ $K \cap K^{\prime}$ and at least one of the two is a member of $\mathscr{G}_{h}$. This means in particular that the boundary faces of the mesh $\mathscr{T}_{h}$ are excluded from $\mathscr{F}_{G}$. We also used $\partial_{n_{F}}$ to denote the derivative in the direction of the normal to $F$.

Coercivity now follows from 58 and 60 as follows

$$
\begin{aligned}
a_{h}\left(u_{h}, u_{h}\right) \geq & \left\|\nabla u_{h}\right\|_{L^{2}(\Omega)}^{2}+\gamma\left\|h^{-\frac{1}{2}} u_{h}\right\|_{L^{2}(\Gamma)}^{2} \\
& -2 C_{T}\left\|\nabla u_{h}\right\|_{L^{2}\left(\mathscr{G}_{h}\right)}\left\|h^{-\frac{1}{2}} u_{h}\right\|_{L^{2}(\Gamma)}+g_{h}\left(u_{h}, u_{h}\right) \\
\geq & c_{G}\left\|\nabla u_{h}\right\|_{\Omega_{\mathscr{T}}}^{2}+\gamma\left\|h^{-\frac{1}{2}} u_{h}\right\|_{L^{2}(\Gamma)}^{2}-2 C_{T}\left\|\nabla u_{h}\right\|_{L^{2}\left(\Omega_{\mathscr{T}}\right)}\left\|h^{-\frac{1}{2}} u_{h}\right\|_{L^{2}(\Gamma)} \\
\geq & \frac{c_{G}}{2}\left\|\nabla u_{h}\right\|_{\Omega_{\mathscr{T}}}^{2}+\left(\gamma-2 C_{T}^{2} c_{G}^{-1}\right)\left\|h^{-\frac{1}{2}} u_{h}\right\|_{L^{2}(\Gamma)}^{2} .
\end{aligned}
$$

Here $C_{T}$ is the constant of the trace inequality 59 and $c_{G}$ is the coercivity constant of the stability estimate (60). We conclude by choosing $\gamma_{0}>2 C_{T}^{2} c_{G}^{-1}$, where the lower bound is independent of the mesh/boundary intersection, but not of the penalty parameter $\gamma_{g}$ in $g_{h}(\cdot, \cdot)$. Error estimates now follow 
in a similar fashion as for the standard Nitsche's method, using 62 and the consistency of the penalty term. One may also show that the conditioning of the system matrix is bounded independently of the mesh/boundary intersection. For further details see [11].

Extension to the other model problems is straightforward. We consider the problem of interface coupling using (35]. In the original paper on cut finite elements [21] the method for meshed subdomains was carried over to the cut element case using piecewise affine elements and weights

$$
w_{1}=K \cap \Omega_{2} /|K|, \quad w_{2}=K \cap \Omega_{1} /|K| .
$$

However, for problems with large contrast $\varepsilon_{\max } / \varepsilon_{\min }$ it is known that thise choice is not stable for arbitrary cuts if the mesh size is not small enough to resolve the contrast. Indeed too large contrast can lead to a phenomenon reminiscent of locking for unfortunate cuts (i.e. if no $H^{1}$-conforming subspace with approximation exists). In case robustness is necessary we instead choose the weights to be $w_{1}=\varepsilon_{2} /\left(\varepsilon_{1}+\varepsilon_{2}\right)$ and $w_{2}=\varepsilon_{1} /\left(\varepsilon_{1}+\varepsilon_{2}\right)$ and $\gamma^{-1}=\gamma_{0} h^{-1} \omega(\varepsilon)$ with $\omega(\varepsilon):=2\left(\varepsilon_{1} \varepsilon_{2}\right) /\left(\varepsilon_{1}+\varepsilon_{2}\right)$ we identify this method as the Nitsche method discussed in [13] 14] which was shown to be stable on unfitted meshes provided a ghost penalty term is added. This additional stabilization term should here have the properties analogous to those for the cut fictitious domain method discussed above, and a typical example is the modification of 61) now acting across the faces of the cut elements on the interface. The resulting method takes the form

$$
\begin{aligned}
& a\left(u_{h}, v_{h}\right)-\left\langle\left\{\left\{\mu \partial_{n} u_{h}\right\}\right\} w,\left[\left[v_{h}\right]\right]\right\rangle_{\Gamma}-\left\langle\left[\left[u_{h}\right]\right],\left\{\left\{\mu \partial_{h} v_{h}\right\}\right\} w\right\rangle_{\Gamma} \\
& +\left\langle\gamma\left[\left[u_{h}\right]\right],\left[\left[v_{h}\right]\right]\right\rangle_{\Gamma}+g_{h}\left(u_{h}, v_{h}\right) \\
& =\left(f, v_{h}\right)_{\Omega}+\left\langle g,\left\langle\left\langle v_{h}\right\rangle_{w}\right\rangle_{\Gamma} .\right.
\end{aligned}
$$

In particular it was proven in [7] that for piecewise affine elements and smooth enough $\Gamma$ there holds

$$
\sum_{i=1}^{2}\left\|\varepsilon_{i} \nabla\left(u_{i}-u_{i, h}\right)\right\|_{\Omega_{i}} \leq C h\|f\|_{\Omega}
$$

where the constant $C$ is independent of $\varepsilon$ and $h$. This result is possible to obtain thanks to the fact that the weights shift the interface term to the side where $\varepsilon_{i}$ is the smallest.

Observe now the resemblance between the formulation (64) and (33). The latter formulation however is not in general robust for large contrast. Indeed regardless of the contrast the constraint will be satisfied equally strongly and we know from the experience of [15] that the trick to obtaining robustness is to relax the control obtained by the multiplier by redefining $\lambda_{h}$. Instead of identifying $\lambda_{h}=\left\{\left\{\varepsilon \partial_{n} u_{h}\right\}\right\}_{w}$ we use that $\left\{\left\{\varepsilon \partial_{n} u_{h}\right\}_{w}=\omega(\varepsilon)\left\{\partial_{n} u_{h}\right\}\right.$ where $\{\cdot\}$ denotes the standard arithmetic average. If we then instead identify $\lambda_{h}=\left\{\partial_{n} u_{h}\right\}$ and introduce the factor $\omega(\varepsilon)$ in the formulation 33 we obtain

$$
\begin{aligned}
a\left(u_{h}, v_{h}\right)+\left\langle\omega(\varepsilon) \lambda_{h}+\gamma\left[\left[u_{h}\right]\right],\left[\left[v_{h}\right]\right]\right\rangle_{\Gamma} & =\left(f, v_{h}\right)_{\Omega}+\left\langle g,\left\langle\left\langle v_{h}\right\rangle\right\rangle_{w}\right\rangle_{\Gamma} \\
\left\langle\left[\left[u_{h}\right]\right], \omega(\varepsilon) \mu_{h}\right\rangle_{\Gamma}-j\left(\lambda_{h}, \mu_{h}\right) & =0
\end{aligned}
$$

where once again $\gamma=\gamma_{0} h^{-1} \omega(\varepsilon)$ and the stabilization operator $j(\cdot, \cdot)$ also must scale as $\omega(\varepsilon)$. This formulation will relax the jump in a similar fashion as 64), but control of $\lambda_{h}$ is sacrificed as $\omega(\varepsilon)$ becomes small.

\section{A numerical example}

We give an example of how the method works in the case of adhesion, with and without contact. To exemplify how the different aspect of adhesion come into play we consider a domain $(0,1) \times(0,1)$ with $u=0$ at $x=0$ and at $y=0$ and with $\partial_{n} u=0$ on other boundaries. The domain is cut by a half circle with radius $r=0.74 ; \varepsilon=2$ on the domain $\Omega_{1}$ containing the origin and $\varepsilon=1 / 2$ on $\Omega_{2}$. The right-hand side is given by

$$
f=\left\{\begin{array}{c}
1 \text { if } y \leq 1 / 2, \\
-7 / 2 \text { if } y>1 / 2 .
\end{array}\right.
$$

We set $\gamma_{0}=100$, used no jump stabilization, and set $\omega(\varepsilon)=1$ but used geometric averages of the type 63.

In Fig 1 we show the solution using the standard Nitsche method 35, in Fig. 2 we show the solution with a cohesive interface with $\kappa=1 / 2$ using [43, and in Fig. 3 we show the solution using a one sided contact condition as in (57). 


\section{Acknowledgements}

The contribution of the first author was supported in part by the EPSRC grants EP/J002313/2 and EP/P01576X/1, the contribution of the second author was supported in part by the Swedish Foundation for Strategic Research Grant No. AM13-0029 and the Swedish Research Council Grant No. 2011-4992.

\section{References}

[1] Pierre Alart and Alain Curnier. A mixed formulation for frictional contact problems prone to Newton like solution methods. Comput. Methods Appl. Mech. Engrg., 92(3):353-375, 1991.

[2] Helio J. C. Barbosa and Thomas J. R. Hughes. The finite element method with Lagrange multipliers on the boundary: circumventing the Babuška-Brezzi condition. Comput. Methods Appl. Mech. Engrg., 85(1):109-128, 1991.

[3] Gabriel R. Barrenechea and Franz Chouly. A local projection stabilized method for fictitious domains. Appl. Math. Lett., 25(12):2071-2076, 2012.

[4] Franco Brezzi and Michel Fortin. Mixed and hybrid finite element methods, volume 15 of Springer Series in Computational Mathematics. Springer-Verlag, New York, 1991.

[5] Erik Burman. Projection stabilization of Lagrange multipliers for the imposition of constraints on interfaces and boundaries. Numer. Methods Partial Differential Equations, 30(2):567-592, 2014.

[6] Erik Burman, Susanne Claus, Peter Hansbo, Mats G. Larson, and André Massing. CutFEM: discretizing geometry and partial differential equations. Internat. J. Numer. Methods Engrg., 104(7):472-501, 2015.

[7] Erik Burman, Johnny Guzman, Manuel A. Sanchez, and Markus Sarkis. Robust flux error estimation of an unfitted Nitsche method for high-contrast interface problems. ArXiv e-prints, February 2016.

[8] Erik Burman and Peter Hansbo. A unified stabilized method for Stokes' and Darcy's equations. $J$. Comput. Appl. Math., 198(1):35-51, 2007.

[9] Erik Burman and Peter Hansbo. Fictitious domain finite element methods using cut elements: I. A stabilized Lagrange multiplier method. Comput. Methods Appl. Mech. Engrg., 199(41-44):2680 2686, 2010.

[10] Erik Burman and Peter Hansbo. Interior-penalty-stabilized Lagrange multiplier methods for the finite-element solution of elliptic interface problems. IMA J. Numer. Anal., 30(3):870-885, 2010.

[11] Erik Burman and Peter Hansbo. Fictitious domain finite element methods using cut elements: II. A stabilized Nitsche method. Appl. Numer. Math., 62(4):328-341, 2012.

[12] Erik Burman, Peter Hansbo, and Mats G. Larson. Augmented Lagrangian finite element methods for contact problems. ArXiv e-prints, September 2016.

[13] Erik Burman and Paolo Zunino. A domain decomposition method based on weighted interior penalties for advection-diffusion-reaction problems. SIAM J. Numer. Anal., 44(4):1612-1638, 2006.

[14] Erik Burman and Paolo Zunino. Numerical approximation of large contrast problems with the unfitted Nitsche method. In Frontiers in numerical analysis-Durham 2010, volume 85 of Lect. Notes Comput. Sci. Eng., pages 227-282. Springer, Heidelberg, 2012.

[15] Erik Burman and Paolo Zunino. Numerical approximation of large contrast problems with the unfitted Nitsche method. In Frontiers in numerical analysis-Durham 2010, volume 85 of Lect. Notes Comput. Sci. Eng., pages 227-282. Springer, Heidelberg, 2012.

[16] Franz Chouly and Patrick Hild. A Nitsche-based method for unilateral contact problems: numerical analysis. SIAM J. Numer. Anal., 51(2):1295-1307, 2013.

[17] Franz Chouly, Patrick Hild, and Yves Renard. Symmetric and non-symmetric variants of Nitsche's method for contact problems in elasticity: theory and numerical experiments. Math. Comp., 84(293):1089-1112, 2015.

[18] Miguel A. Fernández and Mikel Landajuela. Splitting schemes for incompressible fluid/thin-walled structure interaction with unfitted meshes. C. R. Math. Acad. Sci. Paris, 353(7):647-652, 2015. 
[19] Michel Fortin and Roland Glowinski. Augmented Lagrangian methods, volume 15 of Studies in Mathematics and its Applications. North-Holland Publishing Co., Amsterdam, 1983. Applications to the numerical solution of boundary value problems, Translated from the French by B. Hunt and D. C. Spicer.

[20] R. Glowinski and P. Le Tallec. Numerical solution of problems in incompressible finite elasticity by augmented Lagrangian methods. I. Two-dimensional and axisymmetric problems. SIAM J. Appl. Math., 42(2):400-429, 1982.

[21] Anita Hansbo and Peter Hansbo. An unfitted finite element method, based on Nitsche's method, for elliptic interface problems. Comput. Methods Appl. Mech. Engrg., 191(47-48):5537-5552, 2002.

[22] Anita Hansbo and Peter Hansbo. A finite element method for the simulation of strong and weak discontinuities in solid mechanics. Comput. Methods Appl. Mech. Engrg., 193(33-35):3523-3540, 2004.

[23] Mika Juntunen. On the connection between the stabilized Lagrange multiplier and Nitsche's methods. Numer. Math., 131(3):453-471, 2015.

[24] Mika Juntunen and Rolf Stenberg. Nitsche's method for general boundary conditions. Math. Comp., 78(267):1353-1374, 2009.

[25] A Lozinski and M. Fournié. prel: Stabilized lagrange multiplier methods for the approximation of interface stokes' problems on unfitted meshes. In UCL workshop proceedings. Springer Verlag, 2018.

[26] Joachim A. Nitsche. Über ein Variationsprinzip zur Lösung von Dirichlet-Problemen bei Verwendung von Teilräumen, die keinen Randbedingungen unterworfen sind. Abh. Math. Univ. Hamburg, 36:9-15, 1971.

[27] Rolf Stenberg. On some techniques for approximating boundary conditions in the finite element method. J. Comput. Appl. Math., 63(1-3):139-148, 1995. 


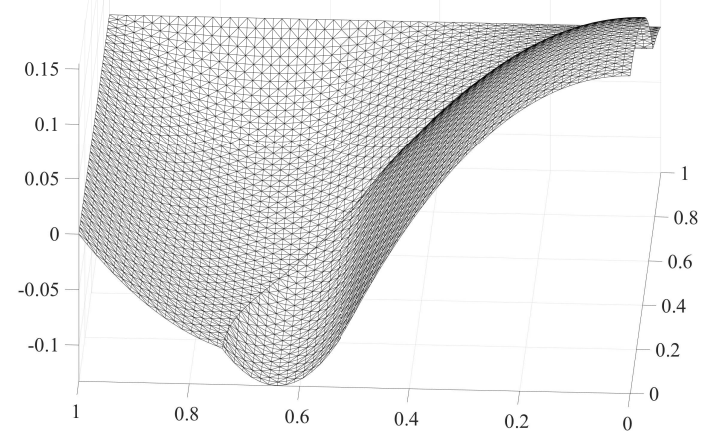

Figure 1: Continuity enforced by Nitsche's method.

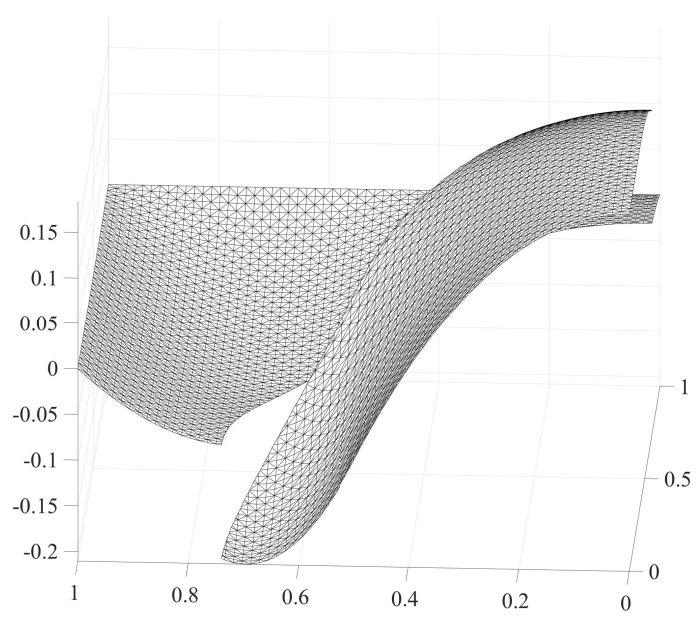

Figure 2: A cohesive interface law enforced by Nitsche's method. 


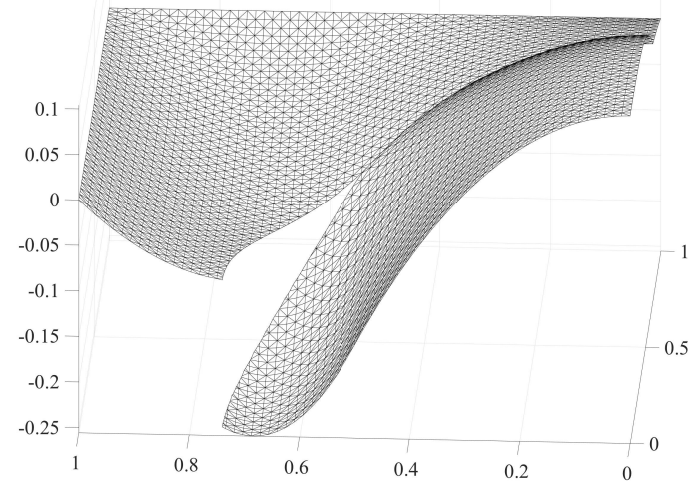

Figure 3: Cohesive interface combined with a contact condition by Nitsche's method. 